\title{
DINÁMICAS URBANAS Y TURISTIFICACIÓN EN EL BARRIO DE ALFAMA (LISBOA): UNA APROXIMACIÓN A LOS CONDICIONANTES HISTÓRICOS Y POLÍTICOS EN EL PROCESO DE AIRBNBIZACIÓN
}

\author{
Jordi Nofre \\ Universidade nova de Lisboa \\ jnofre@fcsh.unl.pt \\ ORCID iD: https://orcid.org/0000-0002-7367-1337 \\ Jorge Sequera \\ UNED \\ jorgesekera@gmail.com \\ ORCID iD: https://orcid.org/0000-0002-8836-425X
}

Recibido: 01/09/2018; Aceptado: 19/06/2019.

Cómo citar este artículo/Citation: Nofre, J. y Sequera, J. (2019). Dinámicas urbanas y turistificación en el barrio de Alfama (Lisboa): una aproximación a los condicionantes históricos y políticos en el proceso de Airbnbización. Estudios Geográficos, 80 (287), e018. https://doi.org/10.3989/estgeogr.201935.015

RESUMEN: Como respuesta a la Gran Recesión (2008-2014) y la consecuente crisis del sistema financiero y de producción nacional, la administración portuguesa adoptó a inicios de esta década el turismo y la rehabilitación urbana como nuevos sectores fundamentales para superar los diferentes impactos socioeconómicos derivados de esta última crisis. Ello ha conllevado procesos de fuerte transformación urbana y social de los barrios históricos de Lisboa. El barrio de Alfama, en el centro de la capital lusa, se ha visto recientemente transformado en una nueva arena financiera glocal para la ejecución de operaciones de especulación inmobiliaria de inversores locales y transnacionales. Este artículo tiene como objetivo analizar la reciente expansión de la turistificación en el Alfama y cómo este nuevo proceso urbano ha interrumpido defacto los procesos de gentrificación y estudentificación iniciados desde finales de los 90s e inicios de la década pasada. En su parte final, el artículo sugiere que la rápida e intensa turistificación y Airbnbización del barrio de Alfama constituye actualmente una de las formas más agresivas de acumulación por desposesión (simbólica y material) y desplazamiento espacial afectando no solamente las clases populares sino también a los primeros gentrificadores del barrio.

PALABRAS CLAVE: Turistificación; gentrificación; desplazamiento espacial; ciudad turística; Lisboa.

\section{URBAN CHANGE AND TOURISTIFICATION IN THE NEIGHBOURHOOD OF ALFAMA (LISBON): AN APPROACH TO THE HISTORIC AND POLITIC FACTORS OF THE PROCESS OF AIRBNBISATION}

ABSTRACT: The Great Recession (2008-2014) and the consequent crisis of both national financial and production system have led the Portuguese administration to adopt tourism and urban rehabilitation as new pivotal sectors to overcome critical crisis-derived impacts on the economy and society. This has involved profound urban and social changes in the central historical quarters of Lisbon. In this sense, the historical quarter of Alfama in Lisbon has recently become the most important urban hotspot in the country for local and transnational real estate investors. By focusing on this historic quarter of Lisbon, this paper examines how recent processes of touristification and Airbnbisation in Alfama have disrupted processes of gentrification and studentification that took place in the quarter since the late 1990s and early 2000s. The paper concludes by suggesting that both urban touristification and Airbnbisation of Alfama emerge today as the most aggressive form of urban accumulation by dispossession and spatial displacement against not only lower classes but also earlier gentrifiers of the quarter.

KEY WORDS: Touristification; gentrification; spatial displacement; tourist city; Lisbon. 


\section{INTRODUCCIÓN}

«El sector de los apartamentos turísticos ha contribuido en gran medida a la rehabilitación urbana del centro de la ciudad de Lisboa, el cual se encontraba muy degradado " ${ }^{1}$ En una entrevista en línea realizada en noviembre de 2017, Francisco Calheiros, presidente de la Confederación Turística Portuguesa, señalaba que tanto las actividades económicas relacionadas con el turismo como la rehabilitación urbana orientada a aumentar la capacidad alojamiento turístico de la capital portuguesa habían emergido definitivamente como sectores fundamentales del llamado " milagro portugués " (The Economist, 2017)². Después de la Gran Recesión y la consecuente crisis de los sistemas financieros y de producción nacionales en un contexto global turbulento, la rápida expansión en los últimos cinco años del sector de los apartamentos turísticos especialmente en áreas centrales de muchas ciudades del sur de Europa ha conllevado la eclosión de protestas y resistencias a la turistificación de la ciudad (Novy y Colomb, 2017), comportando algunos desafíos de primera magnitud en el ámbito de su gobernanza (OECD, 2016). En particular, y análogamente a muchas otras ciudades turísticas tales como Venecia, Roma o Barcelona, la rápida expansión del sector de los apartamentos turísticos en los diferentes barrios históricos del centro de la ciudad de Lisboa (Portugal) ha comportado una marcada tensión alcista en el mercado inmobiliario local (e.g., Barata Salgueiro, 2017), causando intensos procesos de desposesión simbólica y material de las clases populares locales y su consiguiente desplazamiento espacial; procesos socioespaciales que, por otra parte, presentan una serie de rasgos comunes en numerosas ciudades tanto del sur como del norte globales (Janoschka y Sequera, 2016).

En Lisboa (Portugal), la reciente y rápida turistificación de los barrios históricos del centro de la ciudad presenta, ciertamente, algunas singularidades en comparación con otros estudios de caso, tal y como se demostrará en este artículo. En el tradicional barrio popular de Alfama ${ }^{3}$, situado en el corazón de la ciudad y que hasta fechas recientes ha estado habitado mayoritariamente por clases populares, la liberalización del mercado local de alquiler de viviendas en 2012 y la promoción por parte de agentes públicos locales y nacionales de la turistificación de la ciudad - o state-led touristification, en terminología original (Freytag y Bauder, 2018; Pixová y Sladek, 2017) - se han utilizado como estrategias fundamentales de ámbito nacional para la superación de los diferentes impactos socioeconómicos derivados de la última crisis económico-financiera. De hecho, Alfama es actualmente el barrio de Lisboa con la mayor densidad de apartamentos de Airbnb. Según el proyecto municipal Alfama Smart Sustainable District ${ }^{4}$, del total de 3.564 hogares familiares presentes en Alfama según el último Censo de Población de 2011, un total de 937 se encontraban - en Noviembre de 2017 - debidamente registrados en el registro nacional de apartamentos turísticos (Registo Nacional de Alojamento Local, en original) ${ }^{5}$ lo que significa, según Alfama Smart Sustainable District, que existe una capacidad legalmente registrada de 3.836 camas para turistas. Es decir, y de manera meridiana, si Alfama tenía en 2016 alrededor de tan solo 3.000 residentes electores ${ }^{6}$, presentando una disminución del $25 \%$ en tan solo ocho años (JFSM, 2017a, p. 67), en Alfama existe hoy en día 1 cama para turistas por cada habitante.

Aunque algunos autores hablan de la existencia en la actualidad de una intensa interacción entre procesos de gentrificación turística, gentrificación comercial y gentrificación residencial en los barrios históricos del centro de la ciudad de Lisboa (e.g, Barata Salgueiro et al., 2017; Mendes, 2018), este documento pretende ir más allá de este enfoque. Para ello, se tomará como área de estudio el barrio de Alfama, en el centro de la capital lusa, transformado recientemente en uno de los puntos urbanos más importantes del país vecino para la ejecución de operaciones de especulación inmobiliaria de inversores locales y transnacionales relacionadas con el proceso de turistificación urbana de la capital portuguesa. En este artículo se demostrará cómo, por una parte, la reciente expansión de la turistificación y Airbnbización (Richards, 2017) de Alfama ha interrumpido los procesos de gentrificación marginal (Rose, 1984, 1996; Van Criekingen y Decroly, 2003) y primera estudentificación (e.g., Smith, 2008; Hubbard, 2009; Chatterton, 2010; Sage et al., 2012) que, desde finales de los 90s e inicios de la década pasada, formaron parte fundamental de los diferentes programas locales de regeneración urbana y revitalización socioeconómica de los barrios históricos lisboetas.

Por otra parte, y ya en la segunda mitad del artículo, se analizará la rápida e intensa turistificación y Airbnbización del barrio de Alfama. En este sentido, mientras que en muchas ciudades del sur de Europa la turistificación y la gentrificación coexisten simultáneamente, el caso de Lisboa presenta algunas 
diferencias singulares. Lejos de incluso coexistir en una simultaneidad conflictiva como se podría llegar a sugerir, el proceso de turistificación y Airbnbización de Alfama ha implicado la transformación de todo el barrio en un "hotel al aire libre», conllevando un intenso proceso de desplazamiento socio-espacial de la comunidad local, incluidos muchos de aquellos gentrificadores pioneros que llegaron a la zona a finales de la década de 1990 y principios de la de 2000. Para analizar en profundidad cómo esta "interrupción" del proceso de gentrificación marginal y primera estudentificación ha operado en Alfama, se analizará el papel del marco legislativo en la génesis de la turistificación del barrio. Ello llevará a sugerir, ya en la parte final del artículo, que la turistificación de los barrios históricos del centro de Lisboa - y análogamente a otros como Vitória en Porto, La Latina en Madrid o Ciutat Vella en Barcelona - constituye actualmente una de las formas más agresivas de acumulación por desposesión (simbólica y material) y desplazamiento espacial afectando no solamente las clases populares sino también a los primeros gentrificadores de los barrios históricos de la llamada « ciudad turística».
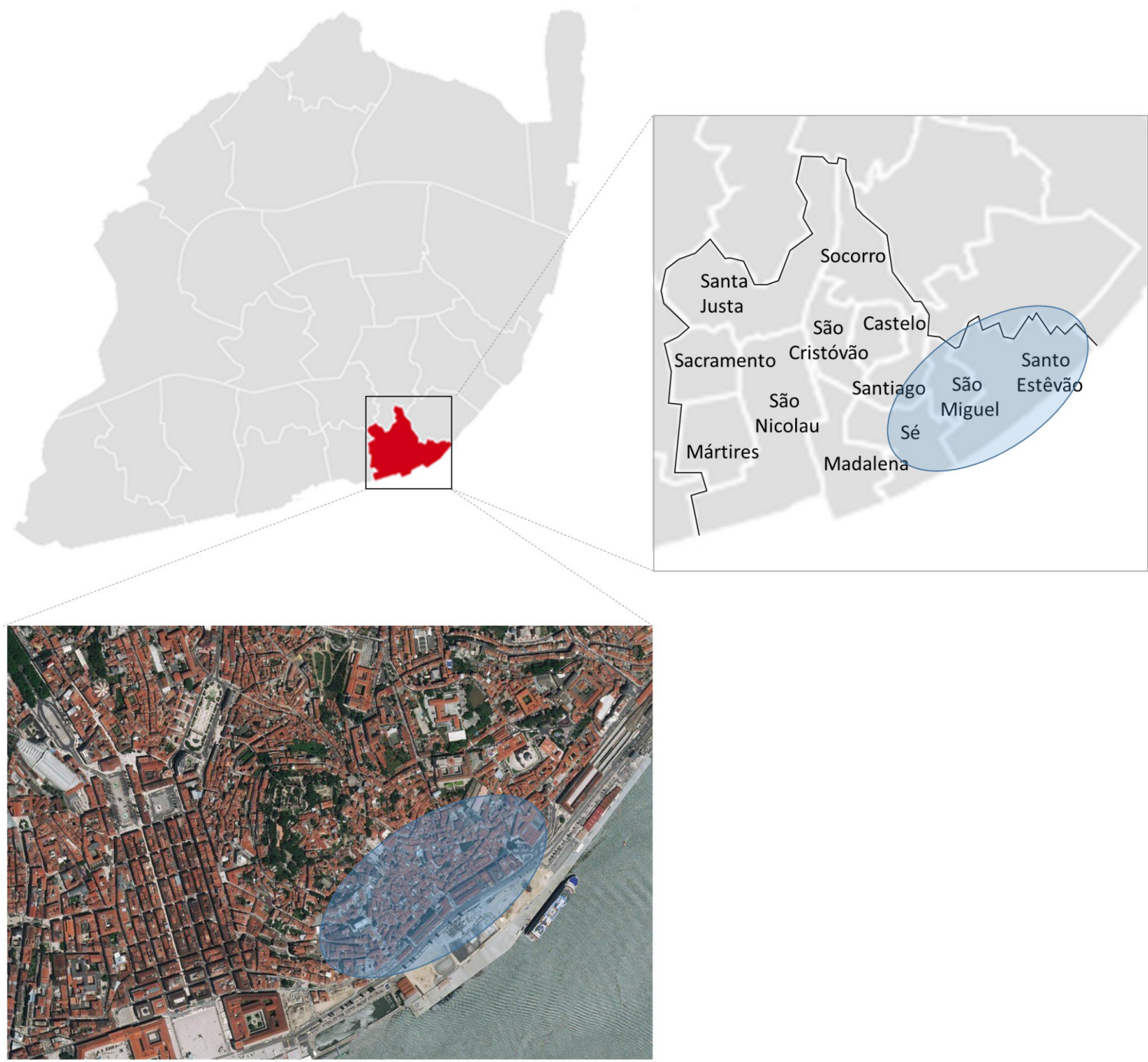

Fuente: Autores. 


\section{Metodología}

La realización de este estudio ha revestido de cierta complejidad. Para la primera parte del artículo, centrada en la evolución urbana del barrio de Alfama hasta 2011, las fuentes de información incluyen (a) Estudios y proyectos centrados en la zona, sobre todo de tipo urbanístico; (b) Legislación urbanística aprobada a lo largo del periodo que atañe este trabajo de investigación; (c) Censos de población del Instituto Nacional de Estadística de Portugal (INE); $y$, finalmente, (d) otras fuentes de información de diversa naturaleza cuya aportación resulta relevante para acabar de perfilar la explicación del proceso de transformación urbana del barrio de Alfama en Lisboa a lo largo de las dos últimas décadas. En relación al Censo de Población, cabe añadir algunas notas técnicas. En primer lugar, los últimos datos censales datan del año 2011, encontrándonos actualmente en plena fase de un periodo intercensal. Ello dificulta sobremanera su uso comparativo con datos relativos a la evolución del proceso de turistificación del espacio urbano considerado en este trabajo. De ahí que a los datos contenidos en el Censo de 2011 del Instituto de Estadística de Portugal se hayan complementado con algunos datos obtenidos de fuentes secundarias para ofrecer al/la lector/a la mayor actualización posible, tal y como se ha procedido, por ejemplo, con el uso del número de electores censados en Alfama, ofrecido por el Ministerio de Administración Interna de Portugal y recogido en el informe Estudo sobre Novas Dinâmicas Residenciais, Económicas e Urbanísticas no Centro Histórico de Lisboa, publicado en se edición revisada en Diciembre de 2017 por las Juntas de Freguesia de Santa Maria Maior, Misericórdia y São Vicente. Precisamente, este informe supra mencionado ha sido de una gran utilidad para la construcción de datos secundarios relativos a la turistificación del barrio de Alfama - y cuyo análisis es presentado en la segunda parte de este artículo -, puesto que estos datos han sido combinados con los datos ofrecidos por compañías de prospección de mercados como Airdna MarketMinder. De manera particular, cabe remarcar que los datos del Sistema de Información Geográfica de Turismo (SIGTUR), y que provienen a su vez del registro nacional de apartamentos turísticos (Registo Nacional de Alojamento Local, en original), son parciales a tenor de la existencia, a fecha de la redacción de este artículo, de numerosos apartamentos turísticos no registrados. A ello cabe sumarle la falta de actualización y la desagregación territorial del todo inadecuada e insuficiente de datos de índole tanto demográfica como turística ofrecidos por diversos or- ganismos y agencias locales y nacionales constituye un reto mayúsculo no solamente para este estudio sino para futuros trabajos en Lisboa el campo de los estudios urbanos. De ahí que, en el caso de cuantificar el stock de apartamentos turísticos existentes en la actualidad, se haya procedido a utilizar la información ofrecida por la plataforma Airdna MarketMinder.

\section{HACIA UNA NUEVA ALFAMA: REGENERACIÓN URBA- NA, ESTUDENTIFICACIÓN Y GENTRIFICACIÓN MARGINAL (2001-2011)}

Entre 1981 y 2011, el municipio de Lisboa experimentó una marcada pérdida de población residente $(-32,2 \%)$, afectando al centro de la ciudad en particular (INE, 2013). Fue durante el período intercensal 1981-1991 cuando la despoblación de las áreas centrales de la ciudad fue más relevante. De hecho, tal vaciamiento de población del centro de la capital lusa fue el resultado de una gama de factores políticos, financieros, sociales y culturales, los cuales incluyen (i) la creciente motorización de la sociedad portuguesa; (ii) la inauguración del primer puente sobre el río Tajo en 1966 y la construcción de las primeras autopistas metropolitanas que rodean a Lisboa; (iii) la (lenta) modernización de los estilos de vida, especialmente en la capital; (iv) una metropolización gradual de las prácticas de ocio de las clases media y media-baja, que se produce con especial relevancia después de la caída del dictadura fascista; y (v) la privatización y liberalización del sistema financiero y bancario portugués en 1984, que tanto aceleró sobremanera los procesos de suburbanización residencial como facilitó el acceso a la financiación de la adquisición de propiedades inmobiliarias (Barata Salgueiro, 1997).

Aunque los gobiernos locales y nacionales formados después del final de la dictadura fascista en 1974 expresaban una notable preocupación por la degradación del stock inmobiliario y la existencia de pobreza urbana en los barrios históricos del centro de la capital portuguesa, no fue sino hasta 1986 que el Ayuntamiento de Lisboa creó la Oficina de Intervención Urbana de Alfama (Gabinete Técnico Local de Alfama, en original). La creación de esta oficina surgió a colación de la declaración de Alfama como Área Crítica para la Recuperación y Reconversión Urbana por parte de la administración local (Decreto № 60/86, de 31 de octubre de 1986). Así, la Oficina de Intervención Urbana de Alfama tuvo como objetivo identificar y examinar los factores negativos que afectaban la degradación de los edificios, proponiendo la necesidad urgente de un programa extraordinario de rehabilitación urbana. 
FIGURA 2

ALFAMA EN 1989. DEGRADACIÓN FÍSICA DE LOS EDIFICIOS
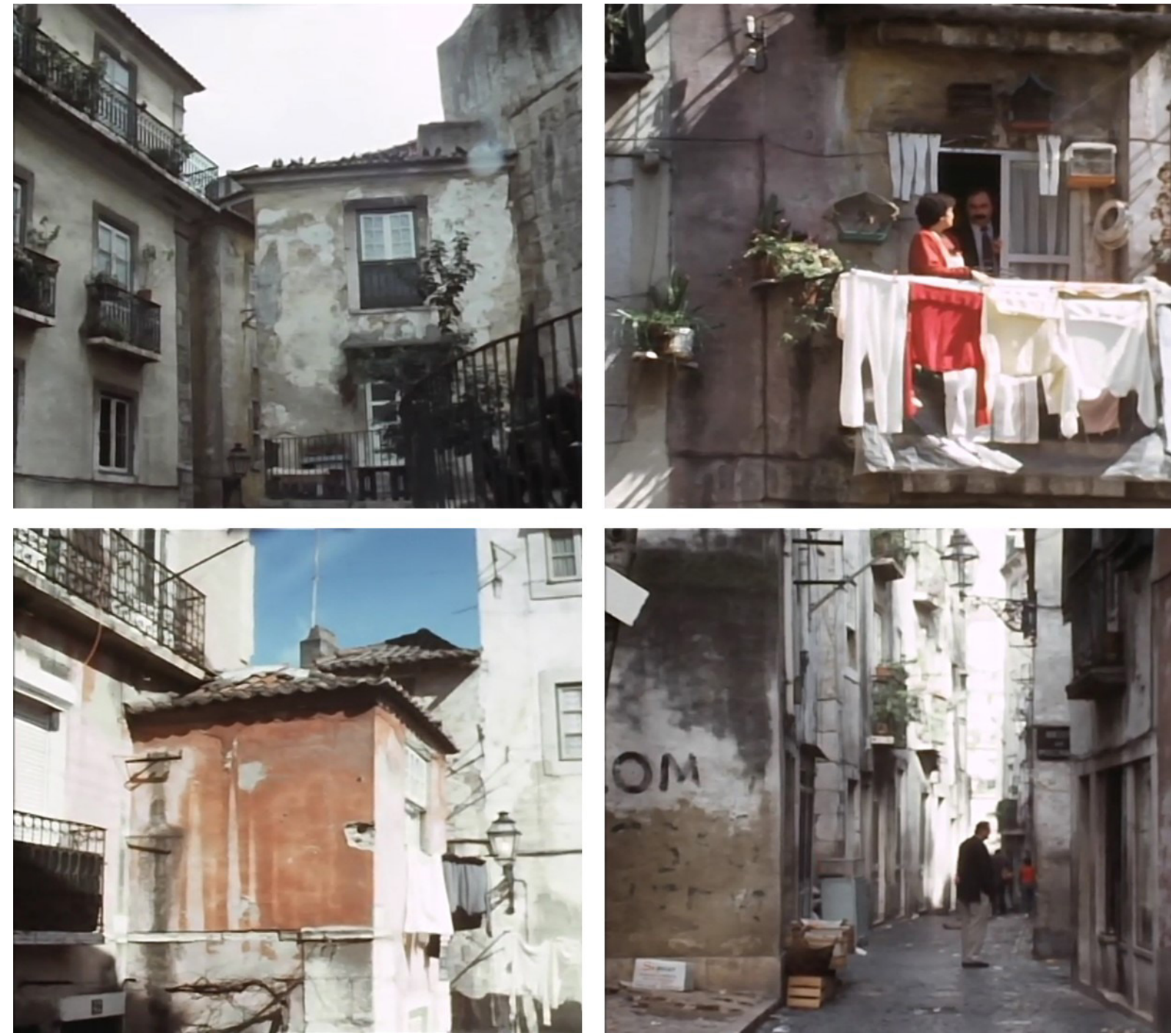

Nota: Secuencia de capturas del documental "Alfama”, del canal 1 de la televisión pública portuguesa RTP, emitido el 5 de septiembre de 1989. Imagen superior izquierda: Degradación del inmueble situado en la torre de la muralla medieval de Lisboa (22:22). Imagen superior derecha: Escena cotidiana de una vecina de la Rua do Vigário, presentando su casa evidentes signos de degradación física (18:51). Imagen inferior izquierda: Casa familiar con evidentes signos de degradación física crítica. Imagen inferior derecha: Escena cotidiana en la Rua de São Miguel, con evidentes signos de degradación tanto del espacio público como de los edificios.

Fuente: RTP (c) 1989. Disponible en línea en: https://arquivos.rtp.pt/conteudos/alfama/\#sthash.9zCB708W.dpbs

Sin embargo, no fue hasta 1997 que la administración local aprobó el Plan de Regeneración Urbana para los barrios de Alfama y Colina del Castillo (Plano de Urbanização do Núcleo Histórico de Alfama e Colina do Castelo, en original; Decreto No. 264/97 del 15 de octubre). De acuerdo con la documentación oficial, los principales objetivos de este plan eran (i) el rejuvenecimiento de la población de ambas áreas, a través de la atracción y fijación de nuevos residentes tales como profesionales jóvenes y adultos jóvenes altamente calificados en sus respectivas áreas profesionales, y (ii) la rehabilitación y la recalificación funcional y estética tanto del edificado como del espacio público, " preservando el espíritu del lugar » (Ib.) o, en otras palabras, construyendo un retroscape (Brown y Sherry, 2003). Adaptando la propuesta de ambos autores para el caso del barrio Alfama, en él los edificios que datan del siglo XVIII e incluso más antiguos, los pequeños bares tradicionales que huelen a tabaco y vino, los colmados tradicionales, las farolas románti- 


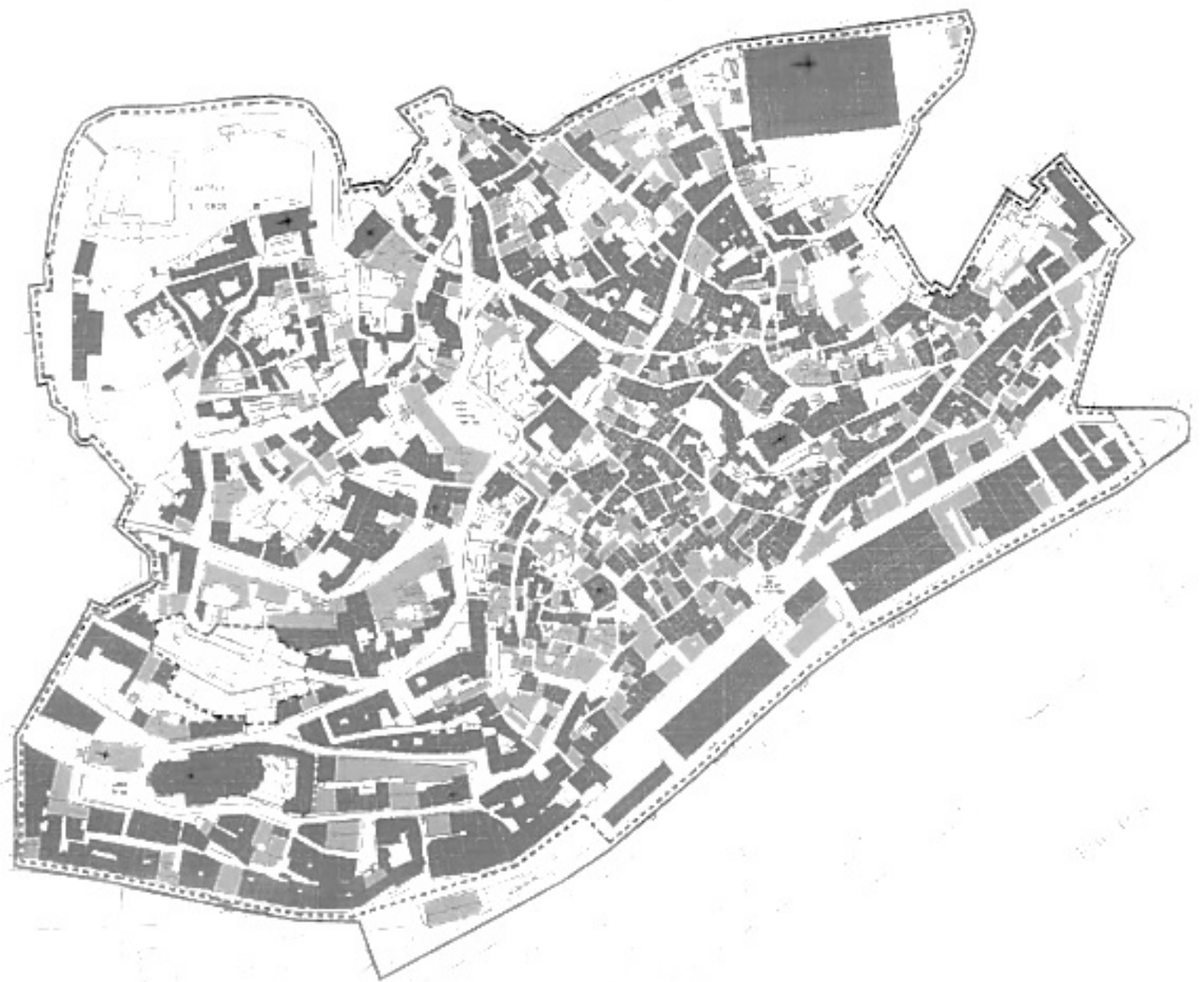

Nota: La variación tonal del color gris mostrado en la figura representa el nivel de degradación del edificado. Los tonos más oscuros corresponden con nivel críticos de degradación.

Fuente: Plano de Urbanização do Núcleo Histórico de Alfama e Colina do Castelo, Câmara Municipal de Lisboa, 1997.

cas y la anciana octogenaria que asoma por la ventana para inspeccionar y controlar « su » calle, constituyen los elementos fundamentales de dicho retroscape, en el que los nuevos residentes se convierten en actores principales de esta « colonización del presente por el pasado » (Belk, 2003, p. 23). En particular, el Plan de Regeneración Urbana de 1997 presentaba la « autenticidad » de Alfama, su «formas de vida tradicionales " y la conformación de un paisaje urbano como una " aldea dentro de la ciudad » (Ib.) como las principales fuerzas motrices para la « atracción atraer al área a nuevos residentes como jóvenes liberales locales y extranjeros y estudiantes universitarios ॥ (Ib.), después de muchas décadas de decadencia social y urbana (França, 2013). De ahí que no sea del todo arriesgado sostener que la promoción por parte de la administración local de un primer proceso de gentrificación y estudentificación del barrio pueda verse como una intervención revanchista (Smith, 1996; Johnsen y Fitzpatrick, 2010; van Eijk, 2010) después de muchas décadas de degradación urbana, miseria, pobreza, exclusión social y guetización.

Los profesionales jóvenes y adultos-jóvenes altamente calificados (especialmente científicos, profesionales liberales e intelectuales) se convirtieron en los principales protagonistas del cambio residencial ${ }^{7}$ en Alfama durante la década de 2000. El descenso de la población residente durante el período intercensal 2001-2011 en el municipio de Lisboa (-3,00 \%) muestra una disminución mucho más pronunciada en el caso de 
Alfama (-20,71\%). Sin embargo, mientras que las tres antiguas freguesias que forman el barrio de Alfama (es decir, Santo Estêvão, São Miguel y Sé) registraron una pérdida significativa de población residente (Santo Estêvão: -26,18\%; São Miguel: -13,81\%; Sé: -21,55\%), la evolución positiva de la población residente de entre 25 y 39 años merece nuestra atención especial.

Un primer análisis de la evolución de la población residente en Alfama durante el periodo intercensal 2001 y 2011 muestra que, si bien el municipio de Lisboa experimentó cierto rejuvenecimiento a pesar de la pérdida de la población total, Alfama registró una disminución muy significativa en residentes de todos los grupos de edad, con excepción de aquellos con edades comprendidas entre 25 y 39 años (ver Tabla 1). Si bien el número de jóvenes que residían en las tres freguesías de Alfama disminuyó durante el período intercensal antes mencionado (Santo Estêvão: $-43,98$ \% para los de 0 a 14 años y -45,38 \% para el grupo de 15-24 años; Miguel: -43.10 \% para el grupo de 0-14 años y $-48,62 \%$ para el grupo de 15-24 años), la población residente de entre 25 y 39 años creció en $+14,16$ \% en Santo Estêvão, en +12,24 \% en São Miguel y en un $+8,66$ en Sé. Estos crecimientos porcentuales son significativamente más altos que los registrados para la población residente en el conjunto Lisboa con edades comprendidas entre los 25 y los 39 años (+5,26\%). Tal y como argumenta Daniel Malet (2013) en su estudio sobre el papel de los estudiantes Erasmus en el proceso de transformación social de Alfama a inicios de la presente década, los datos presentados en la Tabla 1 confirmarían cierto rejuvenecimiento de la población de Alfama durante el período 20012011, el cual estaría estrechamente relacionado con la llegada de estudiantes universitarios y profesionales liberales jóvenes y adultos jóvenes, en lugar de considerarlo como resultado de un hipotético baby-boom.

TABLA 1

EVOLUCIÓN DE LA POBLACIÓN RESIDENTE EN ALFAMA (200-2011), POR GRUPOS ETARIOS

\begin{tabular}{|c|c|c|c|c|c|c|c|c|c|}
\hline & \multicolumn{3}{|c|}{ Número total de residentes } & \multicolumn{3}{|c|}{ De 0 a 14 años } & \multicolumn{3}{|c|}{ De 15 a 24 años } \\
\hline Freguesia & 2001 & 2011 & \%var & 2001 & 2011 & \%var & 2001 & 2011 & \%var \\
\hline Santo Estêvāo & 2047 & 1511 & $-26,18$ & 191 & 107 & $-43,98$ & 238 & 130 & $-45,38$ \\
\hline São Miguel & 1777 & 1531 & $-13,84$ & 232 & 132 & $-43,10$ & 218 & 112 & $-48,62$ \\
\hline Se & 1160 & 910 & $-21,55$ & 88 & 99 & 12,50 & 138 & 62 & $-55,07$ \\
\hline Alfama & 4984 & 3952 & $-20,71$ & 511 & 338 & $-33,86$ & 594 & 304 & $-48,82$ \\
\hline Lisboa & 564657 & 547733 & $-3,00$ & 21287 & 70494 & 231,16 & 44261 & 53507 & 20,89 \\
\hline
\end{tabular}

\begin{tabular}{|c|c|c|c|c|c|c|c|c|c|}
\hline & \multicolumn{3}{|c|}{ De 25 a 39 años } & \multicolumn{3}{|c|}{ De 40 a 64 años } & \multicolumn{3}{|c|}{ Más de 65 años } \\
\hline Freguesia & 2001 & 2011 & $\%$ var & 2001 & 2011 & \%var & 2001 & 2011 & \%var \\
\hline Santo Estêvão & 332 & 379 & 14,16 & 660 & 466 & $-29,39$ & 626 & 429 & $-31,47$ \\
\hline Sāo Miguel| & 343 & 385 & 12,24 & 521 & 468 & $-10,17$ & 463 & 434 & $-6,26$ \\
\hline Sêt & 231 & 251 & 8,66 & 358 & 284 & $-20,67$ & 345 & 214 & $-37,97$ \\
\hline Alfama & 906 & 1015 & 12,03 & 1539 & 1218 & $-20,86$ & 1434 & 1077 & $-24,90$ \\
\hline Lisboa & 112879 & 118815 & 5,26 & 178243 & 173957 & $-2,40$ & 152660 & 130960 & $-14,21$ \\
\hline
\end{tabular}

Fuente: Censos de Población (2001 \& 2011). Instituto Nacional de Estadística de Portugal (INE, 2003, 2013).

TABLA 2

POBLACIÓN RESIDENTE (P. RESID.) EN ALFAMA EN 2001 Y 2011 CON NIVEL DE INSTRUCCIÓN SUPERIOR (PES), Y VARIACIÓN TEMPORAL EN EL PERIODO MENCIONADO

\begin{tabular}{|c|c|c|c|c|c|c|c|}
\hline Freguesia & P. Resid. 2001 & PES 2001 & \% PES 2001 & P. Resid. 2011 & PES 2011 & \% PES 2011 & $\%$ var PES01/11 \\
\hline Santo Estêvāo & 2047 & 125 & 6,11 & 1511 & 263 & 17,41 & 110,40 \\
\hline Sāo Miguel & 1777 & 64 & 3,60 & 1531 & 197 & 12,87 & 207,81 \\
\hline Sê & 1160 & 151 & 13,02 & 910 & 255 & 28,02 & 68,87 \\
\hline Alfama & 4984 & 340 & 6,82 & 3952 & 715 & 18,09 & 110,29 \\
\hline Lisboa & 564657 & 82330 & 14,58 & 547733 & 148423 & 27,10 & 80,28 \\
\hline
\end{tabular}

Fuente: Censos de Población (2001 \& 2011). Instituto Nacional de Estadística de Portugal (INE, 2003, 2013).

TABLA 3

COMPARATIVA ENTRE TOTAL DE POBLACIÓN RESIDENTE, POBLACIÓN EN EDAD ACTIVA, Y NÚMERO DE PROFESIONALES CON NIVEL DE INSTRUCCIÓN SUPERIOR RESIDIENDO EN ALFAMA EN 2011, POR GRANDES GRUPOS ETARIOS

\begin{tabular}{|r|c|c|c|c|c|c|c|c|}
\hline Fregueisa & P. Resid. [PR] & Pobl. Activa [PA] & $<\mathbf{2 4}$ años & $\mathbf{2 5 - 3 9}$ años & $\mathbf{4 0 - 6 4}$ años & Prof.NIS & \% Porf.NIS I PA & \% Prof. NIS / PR \\
\hline Santo Estêvão & 1511 & 734 & 16 & 150 & 59 & 225 & 30,65 & 14,89 \\
\hline Sāo Miguel & 1531 & 660 & 5 & 141 & 43 & 189 & 28,64 & 12,34 \\
\hline Së & 910 & 468 & 3 & 126 & 105 & 234 & 50,00 & 25,71 \\
\hline Alfama & 3952 & 1862 & 24 & 417 & 62 & 648 & $\mathbf{3 4 , 8 0}$ & $\mathbf{1 6 , 4 0}$ \\
\hline Lisboa & 547733 & 260405 & 53507 & \multicolumn{2}{|c|}{292772} & 128080 & $\mathbf{4 9}, \mathbf{1 8}$ & $\mathbf{2 3}, \mathbf{3 8}$ \\
\hline
\end{tabular}

Fuente: Censos de Población (2001 \& 2011). Instituto Nacional de Estadística de Portugal (INE, 2003, 2013). 
De hecho, el extraordinario crecimiento durante el período intercensal 2001-2011 de la población joven y adulta de Lisboa con nivel de instrucción superior (+ $80,28 \%)$ - y el cual se debió en parte a la expansión de la educación superior entre las clases medias-bajas y trabajadoras desde mediados de la década de 2000, entre otros factores socioeconómicos y demográficos - es aún mayor en el caso del barrio de Alfama, con una especial relevancia en el caso de la freguesia de São Miguel (+207.81 \%), 'auténtico corazón' de Alfama. En este barrio tradicionalmente habitado por familias de clase baja, el 34,80\% de los trabajadores activos residentes en Alfama en 2011 presentaban un nivel de instrucción igual o superior a licenciatura. Este porcentaje es aún mayor en la freguesia de Sé $(50,00 \%)$, en donde el porcentaje de población activa altamente calificada de la población total del área es más elevada que en el conjunto del municipio. Tales datos sugerirían que tanto la gentrificación marginal como una primera estudentificación del barrio ciertamente tuvieron lugar durante el período intercensal 2001-2011. Sin embargo, a pesar de este aumento de población adulta-joven residente en Alfama con elevado nivel de instrucción, los datos recientes publicados por el informe Novas Dinâmicas Residenciais, Económicas e Urbanísticas no Centro Histórico de Lisboa, publicado en su edición revisada de Diciembre de 2017 por las Juntas de Freguesia de Santa Maria Maior, Misericórdia y São Vicente, revelan una clara disminución del número de votantes registrados en el barrio de Alfama, presentando un descenso del $25 \%$ en el periodo 2008-2016. A falta de un nuevo recensamiento de la población, previsto para 2021, ello sugeriría la continuación de la pérdida de población en Alfama, situando al barrio en la senda de su vaciamiento poblacional causado, fundamentalmente por un intenso proceso de desplazamiento socio-espacial de la comunidad local - incluidos muchos de aquellos gentrificadores pioneros que llegaron a la zona a finales de la década de 1990 y principios de la de 2000 - y que es provocado por la reciente turistificación y Airbnbización del barrio, tal y como se sostiene en la segunda mitad de este artículo.

\section{EL PAPEL DE LA LEGISLACIÓN EN LA ACTUAL TURISTIFI- CACIÓN DE LISBOA}

La intensidad del proceso de turistificación de la ciudad de Lisboa puede ser entendida de manera más meridiana a tenor de la evolución reciente de algunos indicadores. Por ejemplo, el número de pasajeros de cruceros en Lisboa ha crecido de 164.259 en 2002 a 521.042 en $2017^{8}$, mientras que el número total pasajeros en el aeropuerto de Lisboa ha pasado de 14 millones en 2002 a 22,4 en 2016 y, según los últimos datos recientemente publicados por la prensa nacional, el aeropuerto de Lisboa cerró el año 2017 con un total de 26,7 millones de pasajeros transportados. ${ }^{9}$ Además, al incremento muy significativo de las pernoctaciones en establecimientos hoteleros de la ciudad de Lisboa en los últimos años - pasando de 5.715.176 en 2009 a 9.996.817 en 2015 - cabe sumarle la expansión de hostales (de 97 en 2009 a 229 en 2016) y hoteles (de 93 en 2009 a 167 en 2016) que sobretodo ha encontrado en el área central de la ciudad su mayor área de expansión. ${ }^{10}$ Por otra parte, la comparación entre diversas ciudades del entorno ibérico de la ratio entre el número total de residentes y el número de apartamentos turísticos ofrecidos por la plataforma Airbnb resulta de una especial importancia para vislumbrar aún mejor la intensidad de la turistificación en la capital portuguesa. Mientras que Barcelona presenta actualmente 18.866 apartamentos para 1,6 millones de residentes y Madrid 15.290 apartamentos para 3,1 millones de residentes, Lisboa presenta 14.244 apartamentos para tan solo medio millón de habitantes. Dada la consolidación de Lisboa en el mercado turístico mundial como destino de turismo urbano de primer orden y llegados a este punto del artículo, se antoja como necesario analizar el papel de la administración local y nacional en el proceso de turistificación de la ciudad; es decir, el conjunto de alteraciones de la legislación existente y la implementación de nuevas medidas que, desde el ámbito de lo legislativo, han tenido como objetivo fundamental la promoción de la turistificación de los barrios históricos del centro de Lisboa.

Entre el conjunto de iniciativas legislativas que se comentarán a continuación, debe mencionarse en primer lugar la Nueva Ley de Arrendamiento Urbano (Decreto Ley 6/2006 de 27 de febrero), la cual estableció la primera neoliberalización del arrendamiento urbano al no contemplar, entre muchos otros aspectos, el arrendamiento urbano indefinido para hogares con rentas familiares bajas. Por otra parte, su última alteración mediante Decreto-Ley 31/2012, de 14 de agosto, contenía la introducción de beneficios fiscales para la rehabilitación de edificios, y situaba el 1 de enero de 2017 como fecha límite para el fin de los arrendamientos urbanos antiguos (mayoritariamente con pensionistas de bajos rendimientos que no habían podido huir del centro de la ciudad en los años ochenta y noventa y que vivían en casas antiguas y degradas con un enorme potencial de rehabilitación para su posterior uso turístico). En paralelo, la Câmara 
Municipal de Lisboa aprobó en 2011 la Estrategia de Rehabilitación Urbana de Lisboa 2011-2024, la cual tenía como objetivo principal movilizar una serie de acciones de rehabilitación del stock de edificios sobretodo de los barrios históricos del centro de la ciudad así como la mejora de la calidad del espacio público; ambas acciones debían contribuir a la dinamización del mercado del arrendamiento urbano para vivienda especialmente en el centro histórico de la ciudad. ${ }^{11}$ Para ello, la administración local creó una serie de iniciativas fiscales para la dinamización de la rehabilitación de edificios que tenía como objetivo rebajar la carga impositiva al capital privado. Esta serie de iniciativas las englobó bajo la denominación Programas de Incentivo a la Rehabilitación Urbana. A día de hoy, este programa continua su senda en fomentar la rehabilitación urbana en la ciudad, constando de sub-programas específicos tales como RE9- 9 Ventajas para Rehabilitar Lisboa, Rehabilita Primero y Paga Después y el Instrumento Financiero para la Rehabilitación y Revitalización Urbanas. ${ }^{12}$ Es decir, en pocas palabras, las acciones de apoyo público para la rehabilitación de edificios de viviendas de uso residencial constaban única y exclusivamente de mecanismos de rebajas y exenciones fiscales tanto para los propietarios como para las empresas envueltas en procesos de rehabilitación y promoción.

A su vez, todo este conjunto de acciones legislativas para la de dinamización de la rehabilitación urbana a través de capital privado (y basada exclusivamente en el descenso de la carga impositiva paras las empresas) contó con una nueva iniciativa, que fue la aprobación de un nuevo reglamento denominado Vía Rápida para la Rehabilitación Urbana, el cual fue aprobado en 2013 y tenía como objetivo la simplificación al máximo del proceso administrativo de licencias de obras para la rehabilitación de un edificio de viviendas (Despacho No. 34/P/2013) ${ }^{13}$. Y para mayor simplificación y seguridad jurídica para el capital privado transnacional, Portugal se adhirió al programa europeo Golden Visa Europe en 2012. Sin embargo, existen algunas "lagunas" en la aplicación y/o objetivo real del programa. Ello se desprende de las afirmaciones de Tiago Câmara, de la empresa PTGoldenVisa, en una breve entrevista concedida a World Finance el 5 de abril de $2018 .$.

«El programa de permiso de residencia Golden Visa tiene un gran impacto en la economía portuguesa. Esta es la razón por la cual todos los partidos políticos y el gobierno apoyan constantemente este programa. Hasta el momento, se inyectaron 3.500 millones de euros en Portugal a través del programa Golden Visa; y el 95\% de esta cantidad fue inversión en el sector inmobiliario, [y] el $40 \%$ de todas las transacciones inmobiliarias del año pasado fueron hechas por inversores extranjeros. La mayoría de ellos tenían como objetivo obtener el permiso de residencia. El permiso de residencia de Golden Visa es nuestra gallina de los huevos de oro, y nuestros políticos lo saben. Estamos muy seguros de que el programa seguirá siendo apoyado por nuestro gobierno (...)..»14

Según la organización mundial Transparencia Internacional, los países de origen de los inversores transnacionales que actuaron en 2017 en Portugal a través de su adhesión al programa Golden Visa fueron, de mayor a menor número de inversores que se acogieron al programa, China, Brasil, Rusia, Sudáfrica y Líbano. No es de extrañar, a la luz de los países mencionados, que este programa europeo haya sido recientemente objeto de contundentes críticas por la organización mundial Transparencia Internacional por su connivencia con operaciones de blanqueamiento de capitales realizado por diferentes mafias transnacionales. ${ }^{15}$

En particular, y según las cifras proporcionadas por el Servicio de Extranjería y Fronteras de Portugal, de los 5.717 permisos de residencia otorgados a través del programa Visa Gold entre octubre de 2012 y enero de 2018, 5.397 fueron de inversores inmobiliarios extranjeros quienes - a tenor de las condiciones del mismo programa Visa Gold - adquirieron bienes inmuebles por valor igual o superior a 500.000 euros. Como ejemplo de la importancia del capital transnacional en la reconfiguración del mercado de la vivienda en Lisboa, en el primer semestre de 2016 las inversiones inmobiliarias con fondos transnacionales representaron el $18,3 \%$ de los negocios en el área de rehabilitación urbana de Lisboa, lo que corresponde a la transacción de 909 propiedades por un total de 314 millones de euros (Barata Salgueiro, 2017). Y mientras que nuevos actores transnacionales reconfiguraban el mercado del arrendamiento urbano, la administración nacional aprobaba el segundo reglamento para los apartamentos turísticos (Ley-Decreto 128/2014, de 28 de agosto). Como la primera iniciativa que tuvo lugar en 2008 a través del Decreto Ley no. 39/2008, de 7 de marzo, sobre las nuevas normas legales para la instalación y operación de nuevas unidades de alojamiento turístico -léase aquí "apartamentos turísticos» - no había obtenido un dinamismo como el que era de suponer, este segundo reglamento aprobado en 2014 eximía a los propietarios de los apartamentos turísticos del pago de impuestos especiales, a la vez que se simpli- 
ficaba la tramitación burocrático-administrativa para que cualquier casa para uso principal habitacional pueda ser convertida de manera rápida y simple en un apartamento turístico. Este fue precisamente el paso definitivo para la rápida turistificación de los barrios históricos del centro de Lisboa, como es el caso del barrio de Alfama.

\section{UNA LECTURA GeOgRÁfiCA del PROCESO de AIRBNBI- ZACIÓN DEL BARRIO DE ALFAMA}

Como resultado de lo expuesto en el anterior punto sobre el papel de la legislación en la turistificación del centro de la ciudad de Lisboa, la rápida transformación de vivienda de uso residencial en vivienda de uso turístico temporal ha sido especialmente intensa y rápida en los barrios históricos del centro de Lisboa. Más concretamente, y con el objetivo de visibilizar aún más si cabe el papel crucial de los nuevos players transnacionales de la arena especulativo-inmobiliaria en Lisboa, cabría citar cómo en Alfama los inversores franceses juegan un papel fundamental en la rápida turistificación del barrio, tal y como explica Sofia Rodrigues Nunes, Jefa del Departamento de Vivienda y Planificación Urbana del bufet Gómez-Acebo \& Pombo Abogados en un artículo publicado por la periodista Heather Farmbrough en la prestigiosa revista Forbes, en marzo de 2018:

"Los franceses en particular están comprando casas en los distritos de Chiado, Principe Real, Baixa, Santos, Alfama y Bairro Alto. Buscan palacios antiguos y edificios encantadores, principalmente en las áreas históricas de Lisboa para renovar y convertir en lujosos apartamentos y tiendas carismáticas o hoteles que venden principalmente en el mercado francés. ${ }^{16}$

En Alfama, este interés por parte de nuevos inversores inmobiliarios transnacionales en la adquisición de vivienda para nuevos usos residenciales de lujo y/o turísticos ha conllevado una transformación radical de lo que podríamos denominar topografía social del arrendamiento urbano - en terminología bourdieuana. De manera rápida, en un periodo de tiempo de menos de cinco años, los alquileres de rentas antiguas, así como aquellos indefinidos y a largo plazo (más de cinco años) han dado paso a alquileres de vivienda para uso turístico o para uso residencial temporal inferior a un año, con precios para nada asequibles para las clases populares del barrio (Gago, 2018). Así lo recoge también el estudio publicado a finales de 2017 por la Junta de Freguesia de Santa Maria Maior (en donde Alfama forma parte), Estudio sobre Nuevas Dinámicas Residenciales,
Económicas y Urbanísticas en el Centro Histórico de Lisboa. En su parte dedicada a Síntesis Estratégica y Plan de Acción, el documento pone especial énfasis en creciente presencia, en el centro histórico de Lisboa, de "nuevos usuarios de la ciudad», la "acelerada transformación de los usos de los edificios», "la pérdida de residentes, de negocios tradicionales y de comercio de proximidad», y la «intensificación del proceso de rehabilitación de los edificios» y el consiguiente «incremento significativo de los precios en el mercado de la vivienda [de compra y de alquiler]» (JFSM, 2017b, p. 4).

El impacto económico de la turistificación de los barrios históricos del centro de Lisboa para el mercado laboral local y el erario público no es menor. Según el estudio Qualificação e Valorização do Alojamento Local na Área de Lisboa (2017) Ilevado a cabo por la Asociación de Hoteleria y Restauración de Portugal, la agencia pública Turismo de Portugal, la consultora Quality y coordinado por Hélia Gonçalves Pereira (ISCTE-IUL), a finales del año 2016 el sector de apartamentos turísticos en Lisboa generaba 285,9 millones de euros para las arcas públicas, 5.706 empleos directos y 13.439 empleos indirectos en la ciudad. Sin embargo, y a pesar de su vital importancia en la economía de Lisboa (y de Portugal), la realización de un análisis geográfico de la Airbnización del barrio de Alfama se antoja complicado a tenor de la ausencia clamorosa de datos oficiales y rigurosos sobre dicho proceso de transformación urbana. De hecho, así lo reza el mismo estudio citado en el párrafo anterior, en el que la Junta de Freguesia de Santa Maria Maior se queja amargamente de la falta de información con el grado de actualización y de desagregación geográfica adecuadas para describir fenómenos como la turistificación de su espacio urbano (JFSM, 2017b, p.7). Sin embargo, ello nos impediría realizar una primera lectura geográfica del proceso de turistificación y, más específicamente, de Airbnbización del barrio de Alfama.

Mientras que Alfama representa solamente el $1 \%$ del stock de vivienda residencial de toda la ciudad, el barrio contiene en la actualidad el $10 \%$ de los apartamentos de Airbnb (1.679) existentes en toda la ciudad, según los datos disponibles en el portal Airdna MarketMinder (Junio 2019). De hecho, y tomando como referencia el número de edificios destinados a viviendas residenciales que existían en Alfama según el Censo de 2011 (3.564), el 39,51\% de las viviendas familiares del barrio han sido convertidas en apartamentos turísticos de Airbnb. 
FIGURA 4

MAPA DE LA EVOLUCIÓN DE APARTAMENTOS AIRBNB EN ALFAMA (2015-2017)
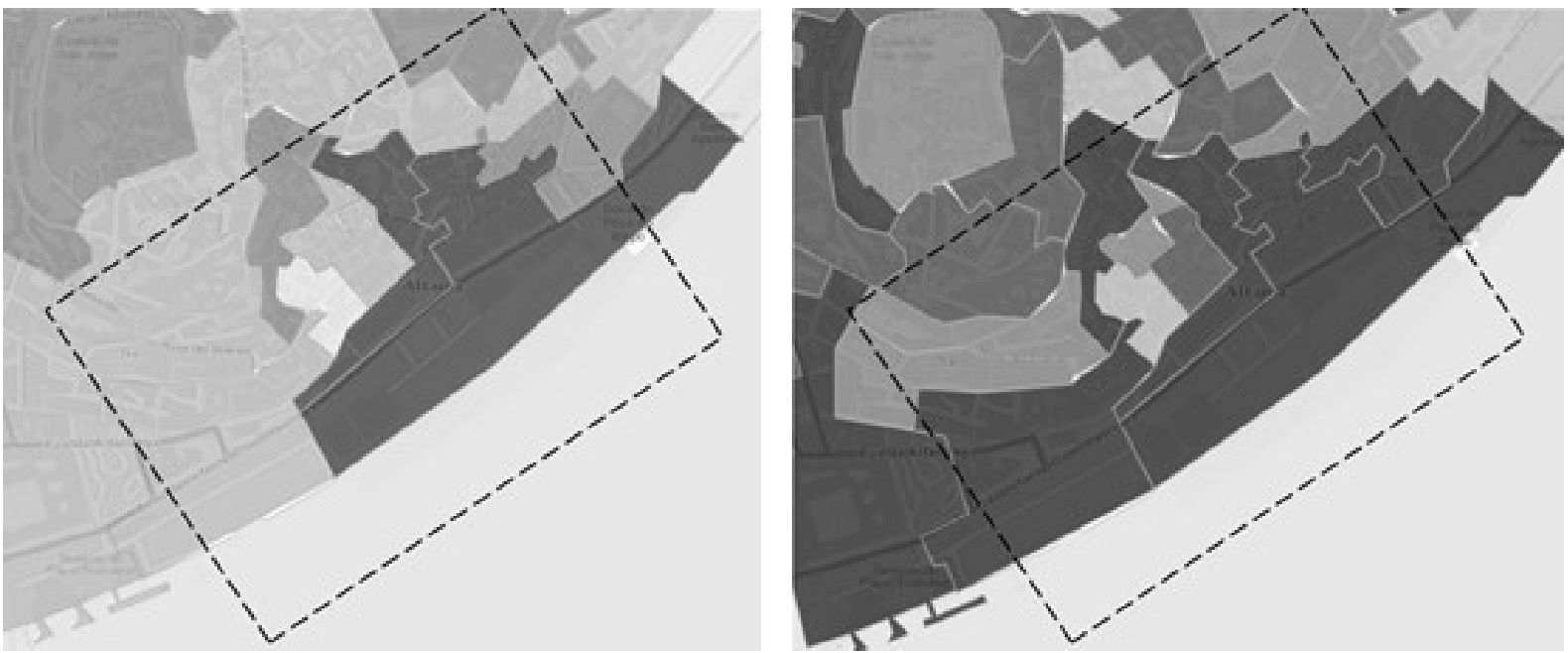

Nota: Para la imagen de la izquierda, los colores oscuros señalan la presencia de más de 60 apartamento turísticos (con un máximo de 90 ) por unidad territorial delimitada en el mapa. Para la imagen de la derecha, los colores oscuros señalan la presencia de más de 80 apartamentos turísticos (con un máximo de 190) en unidades territoriales delimitadas. A pesar de la discrepancia en la construcción de las escalas en lo que se refiere a la lectura de esta cartografía comparativa proveniente de fuentes secundarias, permite entender la fuerte expansión de la Airbnbización en el barrio de Alfama (cuyos límites aproximados vienen señalados en trazo discontinuo).

Fuente: InsideAirbnb. ${ }^{17}$

TABLA 4

TIPOLOGÍA DE ALOJAMIENTO (CASA ENTERA VS. HABITACIÓN PRIVADA) Y TIPOLOGÍA DE LA PROPIEDAD (MULTIPROPIETARIOS VS. PROPIETARIOS PEQUEÑOS), SEGÚN LA DISPONIBILIDAD MÁXIMA DE ALQUILER (365 DÍAS DISPONIBLES)

\begin{tabular}{|r|c|c|c|c|c|}
\hline & Casa Entera & Habitación priv. & Multipropiet. & Propiet. 1 casa & Disponibilidad 365 \\
\hline Lisboa & $77,00 \%$ & $22,00 \%$ & $73,00 \%$ & $27,00 \%$ & $57,00 \%$ \\
\hline Alfama & $94,00 \%$ & $6,00 \%$ & $69,00 \%$ & $31,00 \%$ & $71,00 \%$ \\
\hline
\end{tabular}

Fuente: Airdna MarketMinder (2018).

A tenor de lo mostrado en la Figura 4, junto con el conjunto de observaciones realizados en la primera parte del trabajo de campo observacional, la Airbnbización del barrio de Alfama empezó alrededor de 2011 en su sector más oriental, en la antigua freguesia de Santo Estêvão, y más concretamente en el sector definido por la Rua do Vigário, Rua da Regueira y Rua dos Remédios - muy conocida por la existencia de bares de Fado para turistas y portugueses de clase mediaalta. Ya en 2017, los datos ofrecidos por la plataforma InsideAirbnb coinciden con el trabajo observacional realizado para tal propósito: la Airbnbización del barrio de Alfama fue especialmente intensa a partir de 2016, siendo que en 2017 la práctica totalidad del barrio contenía una densidad de apartamentos elevada, con la excepción del sector comprendido entre Rua da Galé y la iglesia de São Miguel, el tradicional corazón del barrio de Alfama. Sin embargo, a día de hoy tal excepción en la intensidad del proceso de Airbnbización del barrio no existe. En Rua dos Remédios, Rua do Vigário, Rua Adiça y en tantísimas otras calles de
Alfama, ya casi no viven vecinos, tal y como recoge la endo-etnografía (van Ginkel, 1994) iniciada en enero de 2014 y aún en curso por el primer autor y cuyos resultados serán presentados en futuras publicaciones.

\section{CONCLUSIONES}

A lo largo de este artículo se ha analizado el proceso de turistificación y Airbnbización del barrio histórico de Alfama en el centro de la ciudad de Lisboa (Portugal), prestando especial atención tanto al papel de la legislación en dicho proceso de transformación urbana como a una - de entre muchas - de las consecuencias que convierten el caso de Alfama en particular en relación a otros barrios y ciudades del sur de Europa; es decir, cómo la transformación del barrio en nueva arena financiera glocal para la ejecución de operaciones de especulación inmobiliaria de inversores locales y transnacionales en el ámbito del turismo urbano ha comportado un proceso intenso de desplazamiento espacial contra la comunidad local del barrio, incluyendo una interrupción defacto del proceso de gentrificación y estu- 
dentificación iniciados desde finales de los 90s e inicios de la década pasada. De ahí que, en el caso del barrio de Alfama, hablar de "gentrificación turística» o "turismo gentrificador» sea cuanto menos problemático.

Este posicionamiento choca epistemológicamente con la idea de que la gentrificación en la actualidad puede ser entendida como resultado del turismo urbano (Cocola-Gant, 2018), o con las de aquellos que sostienen que la turistificación de las áreas centrales de la ciudad postindustrial puede ser vista como un "vehículo de gentrificación» (Barata Salgueiro et al., 2017), o incluso que el turismo atrae nuevo capital para la gentrificación residencial de áreas centrales de la ciudad (Gravari-Barbas y Guinand, 2017). Si comprendemos el uso del término "gentrificación» enmarcado el sentido "clásico», por el que la gentrificación debería entenderse como una «lucha de clases» (Smith, 1996), observaremos cómo la figura de "el/la turista» - en tiempos de expansión y consolidación de la democratización del turismo para amplias capas de las sociedades posindustriales (si bien no completa) - sitúa al / a la turista como figura no totémica y no homogénea (Quaglieri y Russo, 2010), alejada de un habitus (clase incorporada) del turista.

Por otro lado, la turistificación como estrategia global de acumulación urbana por desposesión simbólica y material (Sequera y Nofre, 2018a) implica que el término «desplazamiento» no solo está relacionado con la gentrificación, sino que afecta al conjunto de la comunidad local - y no solamente a las clases más bajas, como sucede con la gentrificación. Ello ha comportado el aumento de las protestas de los residentes en muchas ciudades del sur de Europa sobre el empeoramiento de la convivencia y la calidad vida en los barrios turísticos (e.g., Novy y Colomb, 2017; Nofre et al., 2018). En Alfama, el malestar no ha eclosionado en la esfera público-mediática como en el caso de Barcelona o Berlín, entre otros casos. Ello se debería, entre otros factores, a los bajos niveles de activismo y participación política en la sociedad portuguesa. Sin embargo, la creciente insatisfacción de los residentes de Alfama se ha expresado en muchas asambleas de residentes organizadas por el presidente de la Junta de Freguesia de Santa Maria Maior, Miguel Coelho, como hemos tenido la oportunidad de verificar a través de nuestra endo-etnografía en Alfama. Sin embargo, estas protestas no solo son expresadas por los residentes de clase baja (principalmente ancianos) de Alfama, sino también por aquellos pioneros de la gentrificación y la estudentificación en Alfama. Mientras que algunas voces y gramáticas de resistencia aparecieron en el barrio visibilizadas a través de pintadas como «Cruceristas Terroristas» o "Fuck Airbnb», el trabajo de campo efectuado permite afirmar que, en el barrio de Alfama, se impone la adaptación forzosa y resignada en el combate contra la turistificación - a diferencia, por ejemplo, de Barcelona o Madrid (Sequera y Nofre, 2018b).

De hecho, la eclosión de la turistificación en Alfama ha permitido crear un espacio común de protesta, debate, reflexión y acción interclasista, es decir, entre las clases populares del barrio y los pioneros gentrificadores, de clase media y media-alta, quienes ven amenazado aquel sueño de vivir en una "aldea dentro de una ciudad cosmopolita y global", tal y como muchos suspiran al ser preguntados sobre sus motivos de intentar quedarse en Alfama a pesar de la profunda transformación de la topografía social del arrendamiento urbano en Alfama. Ya concluyendo este artículo, cabe indicar cómo la turistificación de un espacio urbano determinado no solamente implicaría procesos de desplazamiento espacial sino también la desterritorialización simbólica y sentimental de proyectos de vida individuales y familiares (Davidson y Lees, 2005) lo que, sin duda alguna, escenificaría la urgente necesidad que existe en la actualidad de analizar los impactos - también - en la salud de los individuos (especialmente infancia, juventud y personas mayores) derivados de los procesos de transformación urbana radical como es el caso que nos ha ocupado en este artículo sobre la turistificación y Airbnbización del barrio histórico de Alfama en Lisboa.

\section{AgRADECIMIENTOS}

Este trabajo ha sido realizado en el marco del proyecto "LIKEALOCAL: Efectos socioespaciales de Airbnb. Turismo y Transformación en 4 ciudades de España" (RTI2018-093479-A-I00), financiado por el Ministerio de Ciencia, Innovación y Universidades.

dos-imoveis-convertidos-em-alojamento-localestava-desocupada

2. Veáse el reportaje: "Growing out of it. Portugal cuts its fiscal deficit while raising pensions and wages." The Economist, 01/04/2017. Disponible en: 
https://www.economist.com/news/21719753socialists-say-their-keynesian-policies-areworking-others-fret-about-portugals?fsrc=scn $\% 2$ Ftw\%2Fte\%2Fbl\%2Fed\%2F

3. Para el propósito de este artículo, los autores considerarán el barrio de Alfama como aquel territorio urbano constituido por las antiguas freguesias de Santo Estêvão, São Miguel y Sé.

4. El proyecto municipal Alfama Smart Sustainable District es un proyecto desarrollado conjuntamente por la Facultad de Ciencias y Tecnología de la Universidade Nova de Lisboa y Lisboa E-Nova, agencia municipal de energía y medio ambiente. El proyecto está financiado por la Câmara Municipal de Lisboa, la Junta de Freguesia de Santa Maria Maior, entre otros muchos otros partners relevantes.

5. La plataforma AirDna MarketMinder (Junio de 2019) cifra en 1.679 los apartamentos turísticos de Airbnb en Alfama.

6. Si bien el último Censo de Población fue realizado en 2011, el Ministerio de Administración Interna de Portugal pone a disposición pública y con periodicidad anual el número de electores que existen en las denominadas Juntas de Freguesia, que constituyen el nivel más pequeño de la división administrativa de la República Portuguesa. De ahí que el uso del número de electores pueda ofrecernos una cierta aproximación al número actualizado de población censada en el barrio, si bien no asumimos dicho dato como equivalente a la población residente actual.

7. En este punto se antoja como necesario señalar que las estadísticas proporcionadas por el Instituto Nacional de Estadísticas Portugués (INE) no permiten las referencias cruzadas para distinguir entre los residentes nativos y nuevos de Alfama. Sin embargo, el barrio de Alfama ha estado tradicionalmente habitado por clases de bajos ingresos (Firmino da Costa, A., 1984). mientras que la existencia de un estado de bienestar muy débil en Portugal ha limitado en gran medida la movilidad social ascendente para las generaciones más jóvenes.

8. Datos relativos a la actividad de tráfico de pasajeros del Puerto de Lisboa, según los diferentes informes en línea sobre la actividad portuaria. Disponible en: http://www.portodelisboa.pt/portal/page/portal/PORTAL_PORTO_LISBOA/CRUZEIROS/ESTATISTICAS
9. Para los años anteriores a 2017, véanse los datos disponibles en el portal PORDATA: https:// www.pordata.pt/Portugal/Tr\%C3\%A1fego+de+ passageiros+nos+principais+aeroportos+Lisboa ++Porto+e+Faro-3248. Para 2017, véase: Agência Lusa (2017). Número de passageiros nos aeroportos portugueses sobe $16,4 \%$ em 2017. O Observador, 23/03/2018. Disponible en línea en: https://observador.pt/2018/03/23/numero-depassageiros-nos-aeroportos-portugueses-sobe164-em-2017/

10. Sobre el número de establecimientos hoteleros, véase: https://www.pordata.pt/Municipios/Estab elecimentos+hoteleiros+total+e+por+tipo+de+es tabelecimento-746-4940

11. Para más información, puede consultarse el documento en línea en: http://www.cm-lisboa.pt/ fileadmin/VIVER/Urbanismo/urbanismo/Reabilitacao/estrat.pdf

12. Para más información sobre estos sub-programas, véase: http://www.cm-lisboa.pt/viver/urbanis$\mathrm{mo} / \mathrm{reabilitacao-urbana/programas-municipais-}$ de-incentivo-a-reabilitacao

13. Véase: http://www.cm-lisboa.pt/fileadmin/VIVER/Urbanismo/urbanismo/Reabilitacao/despacho.pdf

14. Disponible en línea en: https://www.worldfinance.com/wealth-management/portugal-launchesnew-investment-fund-path-to-golden-visa

15. Véase: "Golden Visa programmes in Europe pose major corruption risk", Transparency International, Press Release, 05/03/2018. Disponible en línea en: https://www.transparency.org/news/ pressrelease/golden_visa_programmes_in_europe_pose_major_corruption_risk

16. Véase: Farmbrough, H. (2018). "Foreign Investors Boost Property Prices In Lisbon", Forbes, 05/03/2018. Disponible en línea en: https://www.forbes.com/sites/heatherfarmbrough/2018/03/05/foreign-investors-boost-property-prices-in-lisbon/

17. Datos y cartografía disponibles en línea en: https://www.arcgis.com/apps/MapJournal/index.html?appid=950cd79de0fa49788d400dc30a of $2 \mathrm{f} 44$ 


\section{BibliografíA}

Barata Salgueiro, T. (2017). Alojamentos turísticos em Lisboa. Scripta Nova: Revista Electrónica de Geografía y Ciencias Sociales, XXI (578), pp. 1-43. https://doi.org/10.1344/sn2017.21.18587

Barata Salgueiro, T. (1997): “Lisboa, metrópole policêntrica e fragmentada", Finisterra: Revista Portuguesa de Geografia, 63, 179-190. https://doi. org/10.18055/Finis1784

Barata Salgueiro, T., Mendes, L y Guimaraes, P. (2017). Tourism and urban changes. Lessons from Lisbon. En M. Gravari-Barbas y S. Guinand. (Eds.), Tourism and Gentrification in Contemporary Metropolises: International Perspectives (pp. 255-275). Nueva York, EEUU:Taylor \& Francis.

Belk, R. W. (2003). The Sims and the retro future. En S. Brown y J. F. Sherry. (Eds), Time, Space, and the Market: Retroscapes Rising (pp. 35-53). Armonk, NY, EEUU: M.E. Sharpe Publishers.

Brown, S. y Sherry Jr, J. F. (Eds.). (2003). Time, Space, and the Market: Retroscapes Rising. Armonk, NY, EEUU: M.E. Sharpe Publishers.

Chatterton, P. (2010). The student city: an ongoing story of neoliberalism, gentrification, and commodification. Environment and Planning A, 42(3), 509-514. https://doi.org/10.1068/a42293

Cocola-Gant, A. (2018).Tourism gentrification. En Lees, L. y Phillips, M. (Eds), Handbook of Gentrification Studies (281-292). Cheltenham, Reino Unido: Edward Elgar Publishing.

Criekingen, M. van y Decroly, J. M. (2003). Revisiting the diversity of gentrification: neighbourhood renewal processes in Brussels and Montreal. Urban Studies, 40(12), 2451-2468. doi: https://doi.org/1 $0.1080 / 0042098032000136156$

Davidson, M. y Lees, L. (2005). New-build 'gentrification' and London's riverside renaissance. Environment and planning $A, 37(7), 1165-1190$. https:// doi.org/10.1068/a3739

Eijk, G. van (2010). Exclusionary policies are not just about the 'neoliberal city': A critique of theories of urban revanchism and the case of Rotterdam. International Journal of Urban and Regional Research, 34(4), 820-834. https://doi.org/10.1111/ j.1468-2427.2010.00944.x

Farmbrough, H. (2018). Foreign Investors Boost Property Prices In Lisbon. Forbes, 05/03/2018. Re- cuperado de: https://www.forbes.com/sites/ heatherfarmbrough/2018/03/05/foreign-investors-boost-property-prices-in-lisbon/

Firmino da Costa, A. (1984). Entre o cais e o castelo: identidade cultural num tecido social inegualitário. Revista Crítica de Ciências Sociais, (14), 77-108. Recuperado de http://hdl.handle. net/10071/5877

França, J. A. (2013). Lisboa: História Física e Moral. Lisboa, Portugal: Livros Horizonte.

Freytag, T. y Bauder, M. (2018). Bottom-up touristification and urban transformations in Paris. Tourism Geographies, 20(3), 443-460. https://doi.or $\mathrm{g} / 10.1080 / 14616688.2018 .1454504$

Gago, A. (2018). O aluguer de curta duração e a gentrificação turística em Alfama, Lisboa (Tesis de maestria). Instituto de Geografia e Ordenamento do Território, Universidade de Lisboa. Recuperado de http://repositorio.ul.pt/bitstream/10451/32753/19/tm_igotul010887.pdf

Ginkel, R. van (1994). Writing culture from within: Reflections on endogenous ethnography. Etnofoor, 7(1), 5-23. Recuperado de https://www.jstor.org/ stable/25757827

Gonçalves Pereira, H. (coord.) (2017). Qualificação e Valorização do Alojamento Local na Área de Lisboa. Lisboa, Portugal: AHRESP, Turismo de Portugal \& Quality.

Gravari-Barbas, M., y Guinand, S. (2017). Introduction: addressing tourism-gentrification processes in contemporary metropolises. En M. GravariBarbas y S. Guinand. (Eds.), Tourism \& Gentrification in Contemporary Metropolises: International Perspectives (pp. 1-22). New York, EEUU: Taylor \& Francis, pp. 1-22.

Hubbard, P. (2009). Geographies of studentification and purpose-built student accommodation: leading separate lives?. Environment and planning $A$, 41(8), 1903-1923. https://doi.org/10.1068/a4149

INE (2003). XIV Recenseamento Geral da População de Portugal. Lisboa, Portugal: Instituto Nacional de Estatísticas (INE).

INE (2013). XV Recenseamento Geral da População de Portugal. Lisboa, Portugal: Instituto Nacional de Estatísticas (INE). 
Janoschka, M., y Sequera, J. (2016). Gentrification in Latin America: Addressing the politics and geographies of displacement. Urban Geography, 37, 8, 1175-1194. https://doi.org/10.1080/02723638.2015.1103995

Johnsen, S., y Fitzpatrick, S. (2010). Revanchist sanitisation or coercive care? The use of enforcement to combat begging, street drinking and rough sleeping in England. Urban Studies, 47(8), 1703-1723.

JFSM (2017a). Estudo sobre Novas Dinâmicas Residenciais, Económicas e Urbanísticas no Centro Histórico de Lisboa - 1. Enquadramento e Diagnóstico., Lisboa, Portugal: Junta da Freguesia de Santa Maria Maior, Junta da Freguesia da Misericórdia y Junta da Freguesia de Sao Vicente.

JFSM (2017b). Estudo sobre Novas Dinâmicas Residenciais, Económicas e Urbanísticas no Centro Histórico de Lisboa - 2. Síntese estratégica e plano de acção. Lisboa, Portugal: Junta da Freguesia de Santa Maria Maior, Junta da Freguesia da Misericórdia y Junta da Freguesia de São Vicente.

Malet, D. (2013). Procesos de revalorización patrimonial en el barrio de Alfama: el papel de los estudiantes Erasmus en la tematización de la ciudad. Etnográfica, 17 (1), 31-50. https://doi. org/10.4000/etnografica.2532

Mendes, L. (2018). The Panacea of Touristification as a Scenario of Post-Capitalist Crisis. En David, I. (Ed.), Crisis, Austerity, and Transformation: How Disciplinary Neoliberalism Is Changing Portugal (pp. 25-48). New York, EEUU: Lexington Books.

Nofre, J., Giordano, E., Eldrdige, A., J., Martins, J.C., y Sequera, J. (2018). Nightlife, tourism and urban change in the quarter of Barceloneta (20012015)", Tourism Geographies, 20(3), 377-396. https://doi.org/10.1080/14616688.2017.1375972

Novy, J. y Colomb, N. (Eds.). (2017). Protest and Resistance in the Tourist City: Contemporary Geographies of Leisure, Tourism and Mobility. New York, EEUU: Routledge.

OECD. (2016). OECD Tourism Trends and Policies 2016. Paris, Francia: OECD Publishing. https://doi. org/10.1787/tour-2016-en

Pera, G. (2017). Entrevista a Francisco Calheiros: Maioria dos imóveis convertidos em alojamento local estava desocupada. Notícias Ao Minuto, 7/11/2017. Recuperado de https://www.noticiasaominuto. com/economia/895082/maioria-dos-imoveis-convertidos-em-alojamento-local-estava-desocupada
Pixová, M., y Sládek, J. (2017). Touristification and awakening civil society in post-socialist Prague. En J. Novy y N. Colomb. (Eds.), Protest and Resistance in the Tourist City: Contemporary Geographies of Leisure, Tourism and Mobility (pp. 73-89). London, Reino Unido: Routledge.

Quaglieri, A, \& Russo, A. P. (2010). Paisajes urbanos en la época post-turística. Propuesta de un marco analítico. Scripta Nova: Revista Electrónica de Geografía y Ciencias Sociales, 323. Recuperado de http://www.ub.edu/geocrit/sn/sn-323.htm

Richards, G. (2017). Sharing the new localities of tourism. En D. Dredge y S. Gyimóthy. (Eds.), Collaborative Economy and Tourism: Perspectives, Politics, Policies and Prospects (pp. 169-184). Nueva York, EEUU: Springer.

Rose, D. (1984). Rethinking gentrification: beyond the uneven development of Marxist urban theory. Environment and Planning D: Society and Space, 2(1), 47-74. https://doi.org/10.1068/d020047

Rose, D. (1996). Economic restructuring and the diversification of gentrification in the 1980s: A view from a marginal metropolis. En J. Caulfield y L. Peake. (Eds.), City Lives and City Forms: Critical Research and Canadian Urbanism (pp. 131-172). Toronto, Canadá: University of Toronto Press.

Sage, J., Smith, D., y Hubbard, P. (2012). The rapidity of studentification and population change: There goes the (student) hood. Population, Space and Place, 18(5), 597-613. https://doi.org/10.1002/psp.690

Sequera, J. y Nofre, J. (2018a). Shaken, not stirred: New debates on touristification and the limits of gentrification. City, 22(5-6), 843-855. https://doi. org/10.1080/13604813.2018.1548819

Sequera, J. y Nofre, J. (2018b). Urban activism and touristification in southern Europe: Barcelona, Madrid and Lisboa. En J. Roberts y J. Ibrahim. (Eds.), Contemporary Left Wing Activism, vol. 2: Democracy, Participation and Dissent in a Global Context (pp. 88-105). London, Reino Unido: Routledge.

Smith, D. (2008). The politics of studentification and (un)balanced urban populations: Lessons for gentrification and sustainable communities?. Urban Studies, 45(12), 2541-2564. https://doi. org/10.1177/0042098008097108

Smith, N. (1996). The New Urban Frontier: Gentrification and the Revanchist City. New York, EEUU: Psychology Press. 